\title{
MAMÍFEROS DE MÉDIO E GRANDE PORTE EM UM FRAGMENTO DE MATA ATLÂNTICA, MINAS GERAIS, BRASIL ${ }^{1}$
}

\author{
Maressa Rocha do Prado², Ednaldo Cândido Rocha² e Gisele Mendes Lessa del Giudice ${ }^{3}$
}

\begin{abstract}
RESUMO - O grau de ameaça e a importância ecológica dos mamíferos terrestres de médio e grande porte evidenciam a necessidade da busca de informações em inventários e diagnósticos ambientais. Objetivo deste estudo foi inventariar e avaliar a freqüência de ocorrência e riqueza de espécies de mamíferos de médio e grande porte na Estação de Pesquisa, Treinamento e Educação Ambiental (EPTEA) Mata do Paraíso, em Viçosa - MG. A área de estudo foi aleatoriamente percorrida, em busca de evidências indiretas e diretas de mamíferos. Também foram utilizadas armadilhas Tomahawk e fotográficas para o registro e identificação das espécies. Para registrar a freqüência de ocorrência, estabeleceu-se 20 parcelas de 2 x 2 m ao longo de um transecto, as quais foram vistoriadas 29 vezes entre abril de 2005 e abril de 2006. A partir dos dados de freqüência de ocorrência, estimou-se a riqueza de espécies, pelo procedimento Jackknife 1, utilizando o Programa EstimateS. Foram registradas 23 espécies de mamíferos, das quais três estão ameaçadas de extinção: Chrysocyon brachyurus (Illiger, 1815), Leopardus pardalis (Linnaeus, 1758) e Leopardus tigrinus (Schreber, 1775). As espécies silvestres com maior freqüência de registro foram Cerdocyon thous (Linnaeus, 1766), L. tigrinus e L. pardalis. Foi estimada a riqueza de 15 (intervalo de confiança $=0,95$ ) espécies de mamíferos terrestres silvestres para a EPTEA Mata do Paraíso. O presente trabalho mostra que apesar de pequena, a área de estudo desempenha um importante papel na conservação da mastofauna da região de Viçosa - MG.
\end{abstract}

Palavras-chave: mamíferos, riqueza de espécies, pegadas, Mata Atlântica.

\section{MEDIUM AND LARGE-SIZED MAMMAL IN A FOREST FRAGMENT OF ATLANTIC FOREST, MINAS GERAIS, BRAZIL}

\begin{abstract}
The degree of threat and the ecological importance of both medium and large-sized terrestrial mammals point out the necessity of searching for information on inventories and environmental diagnostics. This work aimed to inventory and evaluate the species frequency of occurrence and richness of both medium and large-sized mammals from the Estação de Pesquisa, Treinamento e Educação Ambiental (EPTEA) Mata do Paraíso, Viçosa-MG. The area analyzed was randomly walked in order to find direct or indirect evidences of mammals. It was also used both Tomahawk and photographic traps to register and identify the species. In order to register frequency of occurrence, it was established 20 plots having 2 by $2 \mathrm{~m}$ each one along one transect which were inspected 29 times from April 2005 to April 2006. Based on data of frequency of occurrence, it was estimated the species richness by using Jackknife 1 procedure and the EstimateS program. It was registered 23 species of mammals, from which three are endangered: Chrysocyon brachyurus (Illiger, 1815), Leopardus pardalis (Linnaeus, 1758) and Leopardus tigrinus (Schreber, 1775). The wild species most frequently registered were Cerdocyon thous (Linnaeus, 1766), L. tigrinus e L. pardalis. The degree of richness estimated to the EPTEA on Mata do Paraíso was 15 species of terrestrial wild animals (confidence interval $=0.95)$. This work shows that in spite of the studied area is small it has an important role in the conversation of the mastofauna of Viçosa's neighborhood.
\end{abstract}

Keywords: mammals, species richness, tracks, Atlantic Forest

\footnotetext{
${ }^{1}$ Recebido em 11.09.2006 e aceito para publicação em 19.05.2008.

${ }^{2}$ Programa de Pós-Graduação em Ciência Florestal da Universidade Federal de Viçosa (UFV), Viçosa-MG.. E-mail: < marerp@yahoo.com> .

${ }^{3}$ Departamento de Biologia Animal da UFV. E-mail: <csfboaro@ibb.unesp.br>.
} 


\section{INTRODUÇÃO}

Considerando os mamíferos descritos atualmente, 652 espécies ocorrem em território brasileiro, o que representa aproximadamente $12 \%$ da mastofauna do mundo. Estes números fazem com que o Brasil apresente a maior riqueza de mamíferos em toda a Região Neotropical. Na Mata Atlântica ocorrem cerca de 250 espécies de mamíferos, sendo cerca de $22 \%$ endêmicas a este bioma (REIS et al., 2006).

A Mata Atlântica, por sua localização predominantemente litorânea, foi alvo de forte pressão antrópica desde o descobrimento do Brasil pelos europeus. A extração madeireira, iniciada com o ciclo do pau-brasil e os grandes ciclos da cana-de-açúcar, café, ouro e, mais recentemente, a expansão da pecuária e da silvicultura com espécies exóticas, foram fragmentando a floresta nativa (MENDES, 2004). Esses fatores foram decisivos para gerar o padrão de distribuição das florestas encontrado atualmente na Zona da Mata de Minas Gerais, em pequenos fragmentos secundários (LOPES et al., 2002).

O desmatamento e a fragmentação da MataAtlântica produziram graves conseqüências para a biota nativa, em função da drástica redução de habitats e isolamento genético das populações. Entretanto, deve-se considerar que além da óbvia conseqüência da redução de habitats, muitas espécies de mamíferos são apreciadas como caça ou como animais de estimação e são permanentemente perseguidas em seus habitats naturais (MENDES, 2004).

A região de Viçosa é uma das áreas prioritárias para conservação de mamíferos no Estado de Minas Gerais, sendo classificada como área de alta importância biológica (DRUMMOND et al. 2005). No entanto, estudos sobre mastofauna nessa região ainda são incipientes, possuindo apenas trabalhos precedentes sobre pequenos mamíferos (PAGLIA et al., 1995; LESSA et al., 1999; GONÇALVES e OLIVEIRA, 2004) e primatas (MELO, 1995; PEREIRA, 1995; MORAIS JÚNIOR, 1998; OLIVEIRA et al., 2003; SANTANA, 2006). Sobre os mamíferos de médio e grande porte foi realizado somente um estudo, na Estação de Pesquisa, Treinamento e Educação Ambiental (EPTEA) Mata do Paraíso, o qual apresenta apenas uma lista de espécies para a área (GRIFFITH et al., 1979).

Nestes termos, este estudo foi desenvolvido com o objetivo de inventariar e avaliar a abundância relativa e riqueza de espécies de mamíferos de médio e grande porte na EPTEA Mata do Paraíso, em Viçosa - MG. As informações apresentadas neste trabalho poderão subsidiar futuros planos de manejo e conservação da mastofauna da área.

\section{MATERIAL E MÉTODOS}

\section{1. Área de estudo}

Este estudo foi desenvolvido na Estação de Pesquisa, Treinamento e Educação Ambiental (EPTEA) Mata do Paraíso (ponto central com as seguintes coordenadas geográficas: $20^{\circ} 48^{\prime}$ 'S e $42^{\circ} 51^{\prime} \mathrm{W}$ ). Tratase do maior fragmento de Mata Atlântica presente no município de Viçosa - MG, distante cerca de $5 \mathrm{~km}$ da sede administrativa do município, com área de aproximadamente 384,5 ha (RIBON, 1998), dos quais 194 ha pertencem à Universidade Federal de Viçosa (UFV) e são utilizados para fins educativos e de pesquisa (SILVA et al., 2004).

A área de estudo está inserida na região conhecida como Zona da Mata Mineira e apresenta relevo montanhoso com predominância de solos Latossolo Vermelho-Amarelo, no topo e nas encostas das elevações, e Podzólicos Vermelho-Amarelo Câmbico, nos terraços (REZENDE, 1971; CORRÊA, 1984). A precipitação média anual na região é de $1.221 \mathrm{~mm}$, concentrada entre os meses de outubro a março, e a temperatura média anual é de $19^{\circ} \mathrm{C}$ (VIANELLO e ALVES, 1991). O clima da região, segundo Köppen, é do tipo Cwb, tropical de altitude, com verões quentes e chuvosos e invernos frios e secos (ANTUNES, 1986).

A vegetação arbórea natural da EPTEA Mata do Paraíso foi classificada como floresta secundária residual, composta por vários estádios sucessionais (LEALFILHO, 1992). Porém, no passado, a lavoura de café (Coffea arabica) foi a cultura que mais influenciou a paisagem na região. Após o declínio da cultura do café e abandono dos cafezais, surgiram as pastagens de capim-gordura (Melinis minutiflora), sapé (Imperata brasilienses) e matas secundárias em diferentes estádios de sucessão (VOLPATO, 1994).

\subsection{Inventário das espécies}

Neste estudo, seguiu-se a classificação taxonômica de Wilson e Reeder (2005) e foram considerados mamíferos de médio e grande porte aqueles com peso corporal acima de $1 \mathrm{~kg}$ quando adultos, assim como adotado 
por Chiarello (2000). Desta forma, embora Didelphis aurita (Wied-Neuwied, 1826), Philander frenatus (Olfers, 1818) e Sylvilagus brasiliensis (Linnaeus, 1758) sejam de pequeno porte, foram incluídas neste estudo pois puderam ser seguramente identificadas na área amostrada.

A lista de espécies de mamíferos da área de estudo foi obtida empregando-se os seguintes procedimentos:

1- Toda a área de estudo foi aleatoriamente percorrida, de outubro de 2004 a julho de 2006, em busca de evidências de mamíferos terrestres de médio e grande porte. Tal amostragem incluiu métodos indiretos (identificação de sinais acústicos, pegadas, fezes e pêlos) e direto (visualizações) para o registro das espécies (BECKER e DALPONTE, 1991; BORGES e TOMÁS, 2004).

2- Dez armadilhas do tipo Tomahawk (70,0 x 23,5 x $30,5 \mathrm{~cm}$ ) foram instaladas na trilha do aceiro, que margeia a área pertencente à UFV, a qual passa por ambientes de floresta secundária em diferentes níveis de regeneração, sendo que em alguns trechos havia pastagem em um dos lados. A coleta foi realizada no período de fevereiro a julho de 2006, totalizando 21 noites de amostragem, com $17,14 \%$ de sucesso de captura. Utilizou-se carne ou frutas como iscas e os animais capturados foram identificados, fotografados e liberados logo em seguida.

3- Quatro armadilhas fotográficas do tipo trapacâmera, com sensor infravermelho de detecção de presença e de movimento, foram instaladas individualmente na trilha do aceiro. As armadilhas foram posicionadas no nível do solo, mantendo aproximadamente 4 metros de distância entre elas, e permaneceram ligadas durante todo o dia. O período de coleta foi de agosto de 2005 a maio de 2006, totalizando 198 dias de amostragem.

4- Realizou-se consulta ao acervo da coleção de mamíferos do Museu de Zoologia João Moojen (MZUFV) para verificar a presença de espécimes provindos da EPTEA Mata do Paraíso.

\subsection{Freqüência de registro de pegadas das espécies}

A freqüência de registros de pegadas das espécies de mamíferos terrestres foi obtida utilizando 20 parcelas de 2 x 2 m cada, com uma distância aproximada de 100 m entre elas, estabelecidas ao longo de $2 \mathrm{~km}$ da trilha do aceiro na EPTEA Mata do Paraíso. As parcelas foram preparadas revolvendo o substrato da própria trilha, com o auxílio de enxada, vassoura e rastelo. Elas não foram iscadas e nem umedecidas, visando não alterar o padrão de movimentação dos animais ao longo da área amostrada. A coleta de dados ocorreu de abril de 2005 a abril de 2006, sendo realizadas de uma a três vistorias mensais às parcelas, que totalizaram 29 inspeções e 580 parcelas vistoriadas.

As parcelas eram inspecionadas sempre pela manhã (a partir de 07:30 horas) e cada vistoria demorava cerca de 2 horas. Para evitar recontagem de rastros, na tarde anterior ao dia de coleta de dados, o substrato das parcelas foi revolvido com a enxada e varrida ou rastelada, de forma a apagar as pegadas dos dias anteriores e a tornar o local adequado à impressão de novas pegadas. Uma vez encontradas pegadas, estas foram identificadas (BECKER e DALPONTE, 1991; BORGES e TOMÁS, 2004), fotografadas e, quando necessário, desenhadas para posterior confirmação da identificação.

\subsection{Análise dos dados}

A partir dos dados de presença ou ausência de pegadas de cada espécie nas parcelas, estimou-se a riqueza de espécies, pelo procedimento Jackknife 1 (HELTSHE e FORRESTER, 1983), utilizando o Programa EstimateS versão 7.0 (COLWELL, 2005). O método de registros de pegadas em parcelas previamente preparadas tem se mostrado eficiente para estimar a riqueza de espécies de mamíferos terrestres de médio e grande porte (PARDINI et al., 2003; SCOSS et al., 2004).

A riqueza de espécies total é estima somando a riqueza observada a um parâmetro calculado a partir do número de espécies raras e do número de amostras. Quando todas as espécies observadas ocorrem em mais de uma amostra, a riqueza estimada é igual a observada, ou seja, o método considera que todas as espécies do ambientes foram amostradas (SANTOS, 2003). A estimativa de riqueza de espécies é apresentada seguida de seu intervalo de confiança (IC), ao nível de significância de 95\%.

Avaliou-se também a freqüência de registros das espécies, como indicativo de abundância relativa, dada pelo número total de registros de pegadas de cada espécie nas parcelas, acompanhada de sua representatividade em porcentagem.

R. Árvore, Viçosa-MG, v.32, n.4, p.741-749, 2008 


\section{RESULTADOS E DISCUSSÃO}

\subsection{Inventário das espécies}

Foram registradas 20 espécies de mamíferos silvestres de médio e grande porte e três de pequeno porte, distribuídas em 8 Ordens (Tabela 1), sendo elas: dois didelfídeos, dois pilosas, dois cingulatos, três primatas, sete carnívoros, um artiodáctilo, cinco roedores e um lagomorfo.

As Ordens mais representativas foram Carnivora (30,4\%), Rodentia (21,7\%) e Primates (13,0\%), já as de menor representatividade foram Lagomorpha e Artiodactyla, ambas com 4,3\% cada, seguidas por Cingulata, Pilosa e Didelphimorphia com 8,7\% cada.

O registro por vocalização ocorreu apenas para os primatas e por fezes para os felinos e canídeos. Houve um registro de espinhos de Coendou prehensilis (Linnaeus, 1758) encontrados nas fezes de Leopardus pardalis (Linnaeus, 1758). Utilizando as armadilhas Tomahawk, só houve captura de três espécies: Leopardus tigrinus (Schreber, 1775) $(\mathrm{n}=1)$, D. aurita
( $\mathrm{n}=14)$ e $P$. frenatus $(\mathrm{n}=20)$. Com o uso das armadilhas fotográficas obteve-se fotos de três espécies silvestres (S. brasiliensis, $P$. frenatus e D. aurita), três exóticas (Canis lupus familiaris Linnaeus, 1758) (cachorro doméstico), Bos taurus Linnaeus, 1758 (gado) e Capra sp. (cabra)) e de pessoas.

Consulta ao acervo da coleção mastozoológica do MZ-UFV permitiu registrar Dasyprocta leporina (Linnaeus, 1758), Bradypus variegatus Schinz, 1825 e Dasypus novemcinctus Linnaeus, 1758. As duas primeiras espécies foram identificadas exclusivamente por esse método, sendo que o registro de $D$. leporina no acervo do MZ-UFV é único e datado de 1999. Tal fato pode ter ocorrido em função do histórico de caça predatória em décadas passadas, de forma que sua população pode ter sido muito reduzida ou mesmo extinta na área amostrada, já que não foi obtido registro de $D$. leporina em campo. Além disto, embora $B$. variegatus tenho sido registrada somente através de consulta ao MZ-UFV, moradores e pesquisadores da área confirmam a presença desta espécie na EPTEA Mata do Paraíso.

Tabela 1 - Espécies de mamíferos silvestres registradas na Estação de Pesquisa, Treinamento e Educação Ambiental (EPTEA) Mata do Paraíso, Viçosa - MG

Table 1 - Wild mammals species registered in the Estação de Pesquisa, Treinamento e Educação Ambiental (EPTEA) Mata do Paraíso, Viçosa - MG

\begin{tabular}{|c|c|c|}
\hline Táxon & Nome vulgar & Tipo de Registro \\
\hline \multicolumn{3}{|l|}{ Ordem Didelphimorphia } \\
\hline \multicolumn{3}{|l|}{ Família Didelphidae } \\
\hline Didelphis aurita (Wied-Neuwied, 1826) & gambá de orelha preta & Pg, Vi, Ar, Fo, Ca \\
\hline Philander frenatus (Olfers, 1818) & cuíca de quatro olhos & Ar, Fo \\
\hline \multicolumn{3}{|l|}{ Ordem Pilosa } \\
\hline \multicolumn{3}{|l|}{ Família Bradypodidae } \\
\hline Bradypus variegatus Schinz, 1825 & preguiça & MZ \\
\hline \multicolumn{3}{|l|}{ Família Myrmecophagidae } \\
\hline Tamandua tetradactyla (Linnaeus, 1758) & tamanduá mirim & $\mathrm{Pg}, \mathrm{Ca}$ \\
\hline \multicolumn{3}{|l|}{ Ordem Cingulata } \\
\hline \multicolumn{3}{|l|}{ Família Dasypodidae } \\
\hline Dasypus novemcinctus Linnaeus, 1758 & tatu galinha & Pg, Ca, MZ \\
\hline Euphractus sexcinctus (Linnaeus, 1758) & tatu peba & Pg \\
\hline \multicolumn{3}{|l|}{ Ordem Primates } \\
\hline \multicolumn{3}{|l|}{ Família Cebidae } \\
\hline Callithrix sp. (híbrido) & sagüi, mico, soim & Vo, Vi \\
\hline Callithrix jacchus (Linnaeus, 1758) & sagüi do tufo branco & Vi \\
\hline \multicolumn{3}{|l|}{ Família Pitheciidae } \\
\hline Callicebus nigrifrons (Spix, 1823) & sauá & Vo, Vi \\
\hline \multicolumn{3}{|l|}{ Ordem Carnivora } \\
\hline \multicolumn{3}{|l|}{ Família Canidae } \\
\hline Cerdocyon thous (Linnaeus, 1766) & cachorro do mato & $\mathrm{Pg}, \mathrm{Fe}$ \\
\hline - Chrysocyon brachyurus (Illiger, 1815)*. & _ _ lobo guará _ _ & $\underline{\mathrm{Pg}}, \mathrm{Fe}$ \\
\hline
\end{tabular}

R. Árvore, Viçosa-MG, v.32, n.4, p.741-749, 2008 
Tabela 1 - Cont.

Table 1 - Cont.

\begin{tabular}{|c|c|c|}
\hline Táxon & Nome vulgar & Tipo de Registro \\
\hline \multicolumn{3}{|l|}{ Ordem Carnivora } \\
\hline \multicolumn{3}{|l|}{ Família Procyonidae } \\
\hline Procyon cancrivorus (G. [Baron] Cuvier, 1798) & mão pelada & Pg \\
\hline \multicolumn{3}{|l|}{ Família Mustelidae } \\
\hline Galictis cuja (Molina, 1782) & furão & $\mathrm{Vi}$ \\
\hline \multicolumn{3}{|l|}{ Família Mephitidae } \\
\hline Conepatus semistriatus (Boddaert, 1785) & jaratataca & Pg \\
\hline \multicolumn{3}{|l|}{ Família Felidae } \\
\hline Leopardus pardalis (Linnaeus, 1758)* & jaguatirica & $\mathrm{Pg}, \mathrm{Fe}$ \\
\hline Leopardus tigrinus (Schreber, 1775)* & gato do mato pequeno & $\mathrm{Pg}, \mathrm{Fe}, \mathrm{Ar}$ \\
\hline \multicolumn{3}{|l|}{ Ordem Artiodactyla } \\
\hline \multicolumn{3}{|l|}{ Família Cervidae } \\
\hline Mazama sp. veado & Pg & \\
\hline \multicolumn{3}{|l|}{ Ordem Rodentia } \\
\hline \multicolumn{3}{|l|}{ Família Caviidae } \\
\hline Hydrochoerus hydrochaeris (Linnaeus, 1766) & capivara & Pg \\
\hline Cavia aperea Erxleben, 1777 & preá & $\mathrm{Vi}$ \\
\hline \multicolumn{3}{|l|}{ Família Cuniculidae } \\
\hline Cuniculus paca (Linnaeus, 1766) & раса & Pg \\
\hline \multicolumn{3}{|l|}{ Família Dasyproctidae } \\
\hline Dasyprocta leporina (Linnaeus, 1758) & cutia & MZ \\
\hline \multicolumn{3}{|l|}{ Família Erethizontidae } \\
\hline Coendou prehensilis (Linnaeus, 1758) & ouriço caixeiro & $\mathrm{Pe}$ \\
\hline \multicolumn{3}{|l|}{ Ordem Lagomorpha } \\
\hline \multicolumn{3}{|l|}{ Família Leporidae } \\
\hline Sylvilagus brasiliensis (Linnaeus, 1758) & tapeti, coelho & Pg, Fo, Vi \\
\hline
\end{tabular}

Tipo de Registro: Ar - Armadilha Tomahawk; Ca - Carcaça; Fe - Fezes; Fo - Armadilha fotográfica; MZ - Acervo do Museu de Zoologia João Moojen - UFV; Pe - Pêlo; Pg - Pegada; Vi - Visualização; Vo - Vocalização. * Animais ameaçados de extinção (MMA, 2003; MACHADO et al., 2005).

Type of Record: Ar - Tomahawk Trap; Ca - Carcass; Fe - Feces; Fo - Photographic Trap; MZ - Collection of João Moojen Zoological Museum - UFV; Pe - Hair; Pg - Track; To - Den; Vi - Visualization, Vo - Vocalization. * Endangered animals (MMA, 2003; MACHADO et al., 2005).

Três espécies registradas estão incluídas na lista da fauna brasileira ameaçada de extinção: Chrysocyon brachyurus (Illiger, 1815), L. pardalis e L. tigrinus (MMA, 2003; MACHADO et al., 2005).

A presença de C. brachyurus, uma espécie típica de ambientes com formações vegetais abertas (DIETZ, 1985), possivelmente, é resultado da expansão recente da espécie, em resposta à transformação de áreas de Mata Atlântica em pastagens, zonas agrícolas, campos antrópicos e florestas de Pinus e Eucalyptus. Essas áreas podem ser utilizadas por C. brachyurus, tanto para forragear quanto para descansar (RODRIGUES, 2002; MACHADO et al., 1998).

A grande quantidade de indícios (pegadas e fezes) e evidências diretas (visualização e fotografias) de animais domésticos - cachorros, bovinos, caprídeos e eqüinos - na EPTEA Mata do Paraíso é preocupante dada a sua capacidade de afetar negativamente a fauna silvestre local. Além disto, há relatos da ação de caçadores dentro da EPTEA Mata do Paraíso, pois a caça, mesmo ocorrendo em pequena escala, provoca efeitos sensíveis sobre as densidades populacionais de várias espécies, a qual, juntamente com a fragmentação de habitats, é uma das principais ameaças para a conservação dos mamíferos (COSTA et al., 2005).

\subsection{Freqüência de registro de pegadas e riqueza de espécies}

Registros de pegadas de 13 espécies de mamíferos silvestres terrestres foram obtidos nas parcelas, o que totaliza 76,5\% das espécies listadas neste estudo para a EPTEA Mata do Paraíso e passíveis de identificação por pegadas. Aproximadamente $52 \%$ do total de parcelas apresentaram no mínimo um registro durante o período de coleta. A espécie mais freqüente foi $C$. lupus familiaris, representando 38,4\% dos registros. Entre as espécies

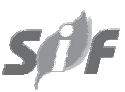

R. Árvore, Viçosa-MG, v.32, n.4, p.741-749, 2008 
silvestres, as de maior número de registros foram Cerdocyon thous (Linnaeus, 1766), L. tigrinus e $L$. pardalis, que apresentaram freqüências de ocorrência de 18,6\%, 9,9\% e 7,9\%, respectivamente. Já as menos freqüentes foram $D$. novemcinctus e Conepatus semistriatus (Boddaert, 1785) com 0,4\% dos registros cada, $C$. brachyurus, com 0,8\% e D. aurita e Tamandua tetradactyla (Linnaeus, 1758), com 1,2\% cada (Tabela 2).

A alta freqüência de registros de pegadas de $C$. lupus familiaris, C. thous, L. tigrinus e L. pardalis está relacionada à limitações do método. O procedimento de registro de pegadas em parcelas ao longo de trilhas tende a gerar elevado número de registros das espécies que percorrem longos trechos em estradas e trilhas, uma vez que um único indivíduo pode deixar suas pegadas marcadas em várias parcelas. Além disto, embora a presença de L. tigrinus tenha sido confirmada na EPTEA Mata do Paraíso por meio da captura de um indivíduo, sua freqüência de registros pode ter sido elevada também em função de suas pegadas serem facilmente confundidas com as de outros gatos pequenos, inclusive com as de Felis catus (Linnaeus, 1758) (gato doméstico) (BECKER e DALPONTE, 1991), que já foi visualizado na área de estudo (LEANDRO MORAES SCOSS, com. pess.). Por outro lado, espécies que possuem o hábito de cruzar as trilhas sem percorrêlas ou percorrendo-as em apenas pequenos trechos, como D. novemcinctus (EDNALDO CÂNDIDO ROCHA, com. pess.), tendem a produzir baixa freqüência de registro, sem que sejam necessariamente menos abundantes.

C. semistriatus foi registrada apenas uma vez na EPTEA Mata do Paraíso, indicando que essa espécie seja realmente rara na área de estudo. Da mesma forma, a baixa freqüência de registros de $C$. brachyurus pode ser explicada pela preferência desta espécie por áreas mais abertas (DIETZ, 1985). Provavelmente, ela tenha utilizado apenas parte da trilha onde uma das margens apresenta vastas áreas abertas, com pastagens, que seriam locais mais adequados à ocorrência dessa espécie.

Fato preocupante é C. lupus familiaris aparecer como a espécie com maior freqüência de registros (38,4\%), pois sua presença pode influenciar na distribuição e abundância da fauna nativa, na medida em que são potenciais transmissores de doenças, têm a capacidade de predar animais silvestres e são competidores diretos por recursos alimentares (BUTLER et al., 2004; CAMPOS, 2004; ROCHA e DALPONTE, 2006).

R. Árvore, Viçosa-MG, v.32, n.4, p.741-749, 2008
Tabela 2 - Espécies, número de registros e freqüência de ocorrência de mamíferos terrestres encontradas na Estação de Pesquisa, Treinamento e Educação Ambiental (EPTEA) Mata do Paraíso, Viçosa MG. * Espécie exótica

Table 2 -Species, number of registers and frequency of occurrence for terrestrial mammals found at the Estação de Pesquisa, Treinamento e Educação Ambiental (EPTEA) Mata do Paraíso, Viçosa - MG. * Exotic species

\begin{tabular}{lcc}
\hline Espécie & No de registros $^{\text {Freqüência de }}$ & $\begin{array}{c}\text { ocorrência (\%) } \\
\text { oconis }\end{array}$ \\
\hline Canis lupus familiaris & 93 & 38,4 \\
Cerdocyon thous & 45 & 18,6 \\
Leopardus tigrinus & 24 & 9,9 \\
Leopardus pardalis & 19 & 7,9 \\
Sylvilagus brasiliensis & 17 & 7,0 \\
Mazama sp. & 15 & 6,2 \\
Euphractus sexcinctus & 9 & 3,7 \\
Cuniculus paca & 6 & 2,5 \\
Procyon cancrivorus & 4 & 1,7 \\
Tamandua tetradactyla & 3 & 1,2 \\
Didelphis aurita & 3 & 1,2 \\
Chrysocyon brachyurus & 2 & 0,8 \\
Conepatus semistriatus & 1 & 0,4 \\
Dasypus novemcinctus & 1 & 0,4 \\
\hline
\end{tabular}

A riqueza de espécies de mamíferos terrestres silvestres de médio e grande porte estimada, utilizando a freqüência de registro obtida nas parcelas, foi 15 (IC $\pm 0,95$ ) (Figura 1), valor próximo ao observado (13 espécies registradas), indicando que a amostragem representou bem esse grupo de mamíferos na área de estudo.

A curva de acumulação de espécies (curva do coletor) teve um acentuado crescimento inicial, mas tendeu à estabilidade a partir de 350 parcelas amostradas, indicando que a amostragem foi suficiente para representar a comunidade de mamíferos terrestres de médio e grande porte da área de estudo (Figura 1).

A metodologia de registro de pegadas em parcelas previamente preparadas mostrou-se eficiente para inventariar e para estimar a riqueza de espécies de mamíferos terrestres de médio e grande porte na EPTEA Mata do Paraíso. Mas, não é um método apropriado para avaliar a abundância relativa das espécies, pois o padrão de deslocamento, o tamanho das áreas de vida e outras características que diferem grandemente entre as espécies de mamíferos terrestres impedem que se assegure a premissa de que o número de pegadas e o número de indivíduos de uma área apresentem o mesmo tipo de correlação em todas as espécies da comunidade (PARDINI et al., 2003). 


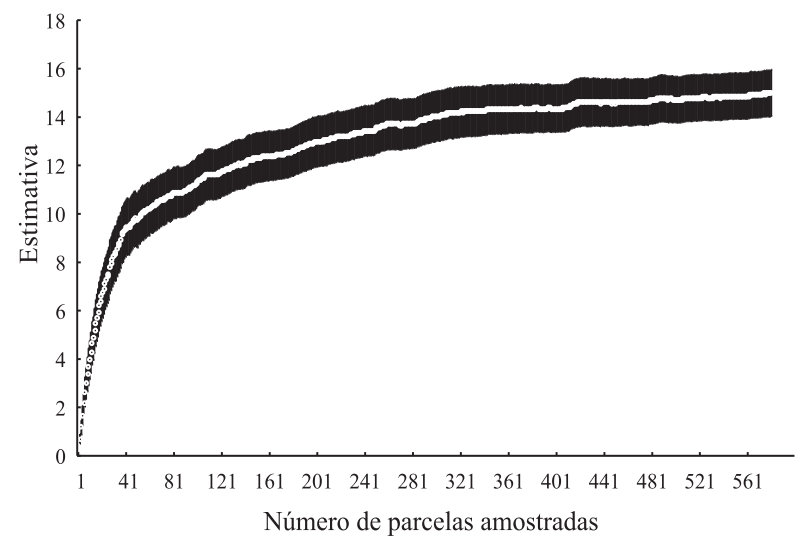

Figura 1 - Estimativa de riqueza de espécies de mamíferos terrestres silvestres de médio e grande porte encontradas na Estação de Pesquisa, Treinamento e Educação Ambiental (EPTEA) Mata do Paraíso, no município de Viçosa - MG. Cada parcela foi considerada uma unidade amostral $(\mathrm{n}=580)$.

Figure 1 - Estimation of species richness of medium and large-sized wild terrestrial mammals found in the Estação de Pesquisa, Treinamento e Educação Ambiental (EPTEA) Mata do Paraíso, in the town of Viçosa-MG, considering each plot as a sample unit $(n=580)$.

\section{CONCLUSÕES}

Os resultados deste estudo mostram que a EPTEA Mata do Paraíso, apesar de pequena, abriga uma riqueza de espécies de mamíferos silvestres de médio e grande porte considerável (20 espécies), incluindo três ameaçadas de extinção: C. brachyurus, L. pardalis e L. tigrinus. Além disto, funciona como área de refúgio num ambiente bastante fragmentado e com muita influência antrópica.

As espécies de mamíferos silvestres que apresentaram maior freqüência de registros de pegadas na EPTEA Mata do Paraíso foram C. thous, L. tigrinus e L. pardalis; já as com menor freqüência foram $D$. novemcinctus, C. semistriatus, C. brachyurus.

A riqueza de espécies de mamíferos terrestres silvestres de médio e grande porte estimada para a EPTEA Mata do Paraíso foi 15 (IC \pm 0,95), valor próximo ao observado (13 espécies registradas).

Por fim, as espécies de mamíferos silvestres presentes na EPTEA Mata do Paraíso não estão totalmente protegidas, tendo em vista a presença de animais domésticos, principalmente de $C$. lupus familiaris que foi a espécie com maior freqüência de registros dentro da área amostrada. Assim, torna-se importante a realização de trabalhos de educação ambiental com os moradores de seu entorno, abordando a importância da EPTEA Mata do Paraíso e o impacto da caça e de animais domésticos sobre ela.

\section{AGRADECIMENTOS}

Ao Conselho Nacional de Desenvolvimento Científico e Tecnológico (CNPq), pela concessão da bolsa de Iniciação Científica; à Universidade Federal de Viçosa, através do Departamento de Biologia Animal, do Museu de Zoologia João Moojen e do Departamento de Engenharia Florestal por ter possibilitado e apoiado a execução deste trabalho; ao Leandro Moraes Scoss e aos revisores anônimos pelas valiosas sugestões.

\section{REFERÊNCIAS BIBLIOGRÁFICAS}

ANTUNES, F.Z. Caracterização Climática do Estado de Minas Gerais. Informe Agropecuário, v. 12, p. 9-13, 1986.

BECKER, M.; DALPONTE, J.C. Rastros de Mamíferos Silvestres Brasileiros: Um Guia de Campo. Brasília, Universidade de Brasília, 1991. 181p.

BORGES, P.A.L.; TOMÁS, W.M. Guia de Rastros e Outros Vestígios de Mamíferos do Pantanal. Corumbá: Embrapa Pantanal, 2004. 139p.

BUTLER, J.R.A.; TOIT, J.T. DU; BINGHAM, J. Free-ranging domestic dogs (Canis familiaris) as predators and prey in rural Zimbabwe: threats of competition and disease to large wild carnivores. Biological Conservation, v.115, p. 369-378, 2004.

CAMPOS, C.B. Impacto de Cães (Canis familiaris) e Gatos (Felis catus) Errantes sobre a Fauna Silvestre em um Ambiente Peri-urbano. 2004. 55f. Dissertação (Mestrado em Ecologia de Agroecossistemas), Universidade de São Paulo, Piracicaba, 2004.

CHIARELLO, A.G. Density and population size of mammals in remnants of Brazilian Atlantic Forest. Conservation Biology, v. 14, n. 6, p.1649-1657, 2000.

R. Árvore, Viçosa-MG, v.32, n.4, p.741-749, 2008 
COLWELL, R.K. 2005. Estimates: Statistical Estimation of Species Richness and Shared Species from Samples. Version 7.0. Disponível em: <http://viceroy.eeb.uconn.edu/ estimates $>$. Acesso em 24.10.2004.

CORRÊA, G.F. Modelo de Evolução Mineralógica da Fração Argila de Solos do Planalto de Viçosa. 1984. $187 \mathrm{f}$. Dissertação (Mestrado em Ciência Florestal), Universidade Federal de Viçosa, Viçosa, 1984.

COSTA, L.P. et al. Conservação de Mamíferos no Brasil. Megadiversidade, v.1, n.1, p.103-112, 2005.

DIETZ, J.M. Chrysocyon brachyurus. Mammalian Species, v.234, p.1-4, 1985.

DRUMMOND, G.M. et al. Biodiversidade em Minas Gerais: um Atlas para sua Conservação. Fundação Biodiversitas, Belo Horizonte, 2005. 222p.

GONÇALVES, P.R.; OLIVEIRA, J.A. Morphological and Genetic Variation Between two Sympatric Forms of Oxymycterus (Rodentia: Sigmodontinae): an Evaluation of Hypotheses of Differentiation within the genus. Journal of Mammalogy, v. 85, n. 1, p. 148-161, 2004.

GRIFFITH, J.J.; CASTRO, P.S.; RIBEIRO, J.C. Levantamentos Básicos e

Recomendações de Manejo para a Reserva Denominada "Mata da Prefeitura”: Relatório Provisório. Departamento de Engenharia Florestal da Universidade Federal de Viçosa, 1979. 86p.

HELTSHE, J.F.; FORRESTER, N.E. Estimating species richness using the Jackknife procedure. Biometrics, v. 39, p. 1-11. 1983.

LEAL-FILHO, N. Caracterização do Banco de Sementes de Três Estádios de uma Sucessão Vegetal na Zona da Mata de Minas Gerais. 1992. 116f. Dissertação (Mestrado em Ciência Florestal). Universidade Federal de Viçosa, Viçosa, 1992.

LESSA, G. et al. Caracterização e monitoramento da fauna de pequenos mamíferos terrestres de um fragmento de mata secundária em Viçosa, Minas Gerais. Bios, v. 7, n. 7, p. 41-49, 1999.
LOPES, W.P. et al. Composição da Flora Arbórea de um Trecho de Floresta Estacional no Jardim Botânico da Universidade Federal de Viçosa (Face Sudoeste), Viçosa, Minas Gerais. Revista Árvore, Viçosa, v.26, n.23, p.339-347, 2002.

MACHADO, A.B.M. et al. Livro Vermelho das Espécies Ameaçadas de Extinção da Fauna de Minas Gerais. Belo Horizonte: Fundação Biodiversitas, 608p., 1998.

MACHADO, A.B.M; MARTINS, C.S.; DRUMMOND, G.M. Lista da Fauna Brasileira Ameaçada de Extinção: incluindo as espécies quase ameaçadas e deficientes em dados. Belo Horizonte: Fundação Biodiversitas, 160p. 2005.

MELO, F.R. Densidade, Tamanho e Composição de grupos de Callicebus personatus (CEBIDAE: PRIMATES) em fragmentos florestais do município de Viçosa, MG. 1995. 29 f. Monografia (Graduação em Ciências Biológicas) - Universidade Federal de Viçosa, Viçosa, 1995.

MENDES, S.L. 2004. Workshop Floresta Atlântica e Campos Sulinos: Grupo de Mamíferos - Documento

Preliminar. Disponível em:

<www.bdt.fat.org.br/workshop/mataatlantica/BR/ rfinais/rt_mamiferos>. Acesso em: 10.10.2004.

MMA - Ministério do Meio Ambiente. Espécies da Fauna Brasileira Ameaçadas de Extinção. Instrução Normativa $\mathrm{n}^{\circ} 3$, de 27 de maio de 2003, publicada no Diário Oficial da União n 101 , de 28 de maio de 2003, Seção 1, p. 88-97.

MORAIS JÚNIOR, M. Aspectos

Ecológicos e Morfológicos de um grupo social de possíveis híbridos de Callithrix (CALLITHRICHIDAE; PRIMATES) em Viçosa, MG. 1998. 25f. Monografia (Graduação em Ciências Biológicas) - Universidade Federal de Viçosa, Viçosa, 1998. 
OLIVEIRA, R.R.; COELHO, A.S.; MELO, F.R. Estimativa de Densidade e Tamanho Populacional de Sauá (Callicebus nigrifrons) em um fragmento de Mata em Regeneração, Viçosa, Minas Gerais, Brasil. Neotropical Primates, v. 11, n. 2, p.91-94, 2003.

PAGLIA, A.P. et al. Heterogeneidade estrutural e diversidade de pequenos mamíferos em um fragmento de mata secundária de Minas Gerais, Brasil. Revista Brasileira de Zoologia, v.12, n. 1, p. 69-79, 1995.

PARDINI, R. et al. Levantamento Rápido de Mamíferos Terrestres de Médio e Grande Porte. In: Métodos de estudos em Biologia da Conservação e Manejo da Vida Silvestre. CULLEN JR., RUDRAN, R.; VALLADARES-PADUA, C. (Orgs.). Editora da Universidade Federal do Paraná, p. 184-201, 2003.

PEREIRA, R.F. et al. Primates from the vicinity of Viçosa, Minas Gerais, Brazil. Neotropical Primates, v. 3, n. 4, p. 171-173, 1995.

REIS, N.R. dos et al. (Eds.). Mamíferos do Brasil. Londrina, 2006. 437p.

REZENDE, S.B. 1971. Estudo de Cronosequência em Viçosa - Minas Gerais. 1971. 71f. Dissertação (Mestrado em Fitotecnia), Universidade Federal de Viçosa, Viçosa, 1971.

RIBON, R. Fatores que influenciam a distribuição da avifauna em fragmentos de Mata Atlântica nas montanhas de Minas Gerais. 1998. $127 \mathrm{f}$. Dissertação (Mestrado em Ecologia, Conservação e Manejo de Vida Silvestre) - Universidade Federal de Minas Gerais, Belo Horizonte, 1998.

ROCHA, E. C.; DALPONTE, J. C. Composição e caracterização da fauna de mamíferos de médio e grande porte em uma pequena reserva de Cerrado em Mato Grosso, Brasil. Revista Árvore, v. 30, n. 4, p. 669-678, 2006.
RODRIGUES, F.H.G. Biologia e

Conservação do lobo-guará na

Estação Ecológica de Águas

Emendadas, DF. 2002. 105f. Tese

(Doutorado em Ecologia). Universidade Estadual de Campinas, Campinas, 2002.

SANTANA, B.E.M.M. Densidade, Tamanho Populacional e Abundância de Primatas em um Fragmento de Mata Atlântica em Minas Gerais. 2006. 24f. Monografia (graduação em Ciências Biológicas), Universidade Federal de Viçosa, Viçosa, 2006.

SANTOS, A.J. Estimativas de riqueza em espécies. In: Métodos de estudos em Biologia da Conservação e Manejo da Vida Silvestre. CULLEN JR.; RUDRAN, R.; VALLADARES-PADUA, C. (Orgs.). Editora da Universidade Federal do Paraná, p. 19-41, 2003.

SCOSS, L.M. et al. Uso de Parcelas de Areia para o Monitoramento de Impacto de Estradas sobre a Riqueza de Espécies de Mamíferos. Revista Árvore, Viçosa, v. 28, n. 1, p. 121-127, 2004.

SILVA, C.T. et al. Avaliação temporal da florística arbórea de uma floresta secundária no município de Viçosa, MG. Revista Árvore, v. 28, n. 3, p.429-441, 2004.

VIANELLO, R.L.; ALVES, A.R. Meteorologia Básica e Aplicações. Viçosa, UFV, Imprensa Universitária, 449p., 1991.

VOLPATO, M.M.L. Regeneração Natural em uma Floresta Secundária no Domínio de Mata Atlântica: uma Análise Fitossociológica. 1994. 123f. Dissertação (Mestrado em Ciência Florestal), Universidade Federal de Viçosa, Viçosa, 1994.

WILSON, D.E.; REEDER, D.A. (Eds.). Mammal species of the World: a taxonomic and geographic reference. Third Edition, v. 1 e 2, The Johns Hopkins University Press, 2142p., 2005. 